\title{
The Association of Chronic Kidney Disease and Metabolic Syndrome with Incident Cardiovascular Events: Multiethnic Study of Atherosclerosis
}

\author{
Subhashish Agarwal, ${ }^{1}$ Michael G. Shlipak, ${ }^{2}$ Holly Kramer, ${ }^{3}$ Aditya Jain, ${ }^{4}$ \\ and David M. Herrington ${ }^{1}$ \\ ${ }^{1}$ Cardiology Section, Wake Forest School of Medicine, Winston-Salem, NC 27157, USA \\ ${ }^{2}$ General Internal Medicine Division, San Francisco VA Medical Center and the University of California San Francisco, \\ San Francisco, CA 94143, USA \\ ${ }^{3}$ Department of Preventive Medicine and Division of Nephrology and Hypertension, Loyola University Medical Center, \\ Maywood, IL 60153, USA \\ ${ }^{4}$ Department of Radiology, John Hopkins University, Baltimore, MD 21205, USA
}

Correspondence should be addressed to Subhashish Agarwal, sa1972@gmail.com

Received 21 April 2011; Accepted 22 June 2011

Academic Editor: Anjali Arora

Copyright (C) 2012 Subhashish Agarwal et al. This is an open access article distributed under the Creative Commons Attribution License, which permits unrestricted use, distribution, and reproduction in any medium, provided the original work is properly cited.

\begin{abstract}
Background. There is an association between chronic kidney disease (CKD) and metabolic syndrome (MetS). We examined the joint association of CKD and MetS with incident cardiovascular (CVD) events in the Multiethnic Study of Atherosclerosis (MESA) cohort. Methods. We analyzed 2,283 Caucasians, 363 Chinese, 1,449 African-Americans, and 1,068 Hispanics in the MESA cohort. CKD was defined by cystatin C estimated glomerular filtration rate $\leq 60 \mathrm{~mL} / \mathrm{min} / 1.73 \mathrm{~m}^{2}$ and MetS was defined by NCEP criteria. Cox proportional regression adjusting for age, ethnicity, gender, study site, education, income, smoking, alcohol use, physical activity, and total and LDL cholesterol was performed to assess the joint association of CKD and MetS with incident CVD events. Participants were divided into four groups by presence of CKD and/or MetS and compared to the group without CKD and MetS $\left(\mathrm{CKD}^{-} / \mathrm{MetS}^{-}\right)$. Tests for additive and multiplicative interactions between CKD and MetS and prediction of incident CVD were performed. Results. During follow-up period of 5.5 years, 283 participants developed CVD. Multivariate Cox regression analysis demonstrated that CKD and MetS were independent predictors of CVD (hazard ratio, 2.02 for CKD, and 2.55 for MetS). When participants were compared to the $\mathrm{CKD}^{-} / \mathrm{MetS}^{-}$group, adjusted $\mathrm{HR}$ for the $\mathrm{CKD}^{+} / \mathrm{MetS}^{+}$group was 5.56 (95\% CI 3.72-8.12). There was no multiplicative interaction between CKD and MetS $(P=0.2)$; however, there was presence of additive interaction. The relative excess risk for additive interaction (RERI) was $2.73, P=0.2$, and the attributable portion (AP) was 0.49 (0.24-0.74). Conclusion. Our findings illustrate that the combination of CKD and MetS is a strong predictor of incident clinical cardiovascular events due to presence of additive interaction between CKD and MetS.
\end{abstract}

\section{Introduction}

A large percentage of the US population (10\%) suffers from chronic kidney disease (CKD) [1] which is associated with metabolic syndrome [2-4]. Metabolic syndrome (MetS) is a construct of physical and laboratory anomalies that confers a higher risk for diabetes mellitus, cardiovascular events and mortality. The National Cholesterol Education Program Adult Treatment Panel (ATP III) criteria define MetS as having at least three of the following: abdominal or central obesity; high triglyceride levels; low high-density lipoprotein (HDL) cholesterol; hyperglycemia; hypertension [5], which has high prevalence in the US [6].

Both CKD [7-9] and MetS [10, 11] have been shown to be independently associated with increased cardiovascular events and mortality, and studies suggest that CKD and MetS are associated with each other as well [2-4]. The increased cardiovascular risk of kidney disease is partly explained by 
an increased burden of traditional cardiovascular risk factors, such as abnormalities in serum lipid concentrations and distribution (elevated triglycerides and lower high-density lipoprotein), diabetes mellitus, and hypertension [2, 12, 13] which are also part of the MetS construct. There is evidence to suggest that CKD progression and associated adverse CVD outcomes are related to severe vitamin D deficiency. The recent findings of vitamin $\mathrm{D}$, being a modulator of both insulin resistance [14] and the renin-angiotensin system [15] and the implication of the renin-angiotensin system in local pancreatic islet structure and function $[16,17]$, suggest that perhaps renal dysfunction and MetS may share common pathological pathways. This overlap in associated risk factors combined with the potential modifying effect of impaired renal function raises the question about whether the cooccurrence of both conditions would augment or attenuate the anticipated risk based on the effect of the two risk factors individually. The purpose of this study is to understand the joint associations of the two conditions with CVD events in a multiethnic population which could lead to improvements in risk stratification and determine whether participants with both conditions should be specifically targeted for more aggressive and early risk factor interventions. Because cystatin $\mathrm{C}$ appears to be more sensitive in detecting mild to moderate decrease in glomerular filtration rate [18, 19], shows strong associations with incident cardiovascular events [20] and all cause mortality [20, 21], and is not affected by age, gender, ethnicity, or muscle mass, this study focused on CKD defined by reduced GFR using a cystatin Cbased estimating equation [22].

\section{Materials and Methods}

2.1. Study Population. The Multiethnic Study of Atherosclerosis (MESA) design has been previously described [23]. Briefly, MESA is a prospective cohort study that began in July 2000 to investigate the prevalence, correlates, and progression of subclinical CVD. The study included 6814 men and women aged 45-84 years old recruited from 6 US communities (Baltimore, MD; Chicago, IL; Forsyth County, NC; Los Angeles County, CA; northern Manhattan, NY; St. Paul, MN). MESA cohort participants were 38\% Caucasian $(n=2622), 28 \%$ African-American $(n=1893)$, $22 \%$ Hispanic $(n=1496)$, and $12 \%$ Chinese $(n=803)$. Individuals with a history of physician-diagnosed myocardial infarction, angina, heart failure, stroke, or transient ischemic attack, or who had undergone an invasive procedure for CVD (coronary artery bypass graft, angioplasty, valve replacement, pacemaker placement, or other vascular surgeries) were excluded from the study at baseline (2000-2002). After excluding participants with missing data on serum cystatin C $(n=58)$ and covariates $(n=1143)$, we had 5,613 participants at baseline with complete data on serum cystatin C, Mets, and covariates of interest. This study was approved by the Institutional Review Boards of each study site and written informed consent was obtained from all participants.

2.2. Laboratory Measures and Data Collection. Medical history, anthropometric measurements, and laboratory data for the present study were taken from the first examination of the MESA cohort (July 2000-August 2002). Information about age, sex, ethnicity, and medical history were obtained by questionnaires. Resting blood pressure was measured using the Dinamap monitor PRO 100 (Critikon, Tampa, Fla, USA) automated oscillometric device. Three measurements were obtained at 1-min intervals with the subject in the seated position with back and arm supported after 5 min of rest with an appropriate-sized cuff, with the cuff at the level of the heart, using a standardized protocol. The average of the second and third measurements was recorded as the resting blood pressure. Hypertension was defined as a systolic blood pressure $\geq 140 \mathrm{~mm} \mathrm{Hg}$, a diastolic blood pressure $\geq 90 \mathrm{~mm} \mathrm{Hg}$, or currently taking medications for blood pressure control [24]. Smoking use was defined as never, former, and current smokers. Smoking ever is defined as $\geq 100$ cigarettes in one's lifetime; current is defined as having smoked a cigarette in the last 30 days. Diabetes was defined as a fasting glucose $\geq 126 \mathrm{mg} / \mathrm{dL}$ or use of insulin or hypoglycemic medications. Plasma lipids (HDL cholesterol, triglycerides, and total cholesterol) were measured from blood samples obtained after a 12-hour fast and measured using a standardized kit (Roche Diagnostics). LDL cholesterol was calculated with the Friedewald equation [25]. Cystatin C was measured from frozen sera at a central laboratory (University of Vermont, Colchester, Vt, USA) using a BNII nephelometer (Dade Behring Inc, Deerfield, Ill, USA) and a particle-enhanced immunonephelometric assay (N Latex Cystatin C; Dade-Behring) [26]. The analytical coefficient of variation for this assay is $2.5 \%$.

Chronic kidney disease was defined as cystatin $\mathrm{C}$ derived glomerular filtration rate $\leq 60 \mathrm{~mL} / \mathrm{min} / 1.73 \mathrm{~m}^{2}$ using the formula derived and validated by Stevens et al. [22] $\left(\mathrm{eGFR}_{\mathrm{cysC}}=\right.$ $76.7 *$ cystatin $\left.\mathrm{C}^{-1.19}\right)$. The National Cholesterol Education Program/Adult Treatment Panel (NCEP ATP III) [5, 27] definition was used to classify participants having MetS in the MESA cohort. Three of five components are required for diagnosis. (1) Waist circumference $\geq 102 \mathrm{~cm}$ : men, $\geq 88 \mathrm{~cm}$ : women, (2) hypertension $\geq 130 \mathrm{~mm} \mathrm{Hg}$ systolic or $\geq 85 \mathrm{~mm} \mathrm{Hg}$ diastolic or use of medications for hypertension, (3) fasting blood glucose $\geq 100 \mathrm{mg} / \mathrm{dL}$ or treatment for impaired fasting glucose, (4) triglycerides $\geq 150 \mathrm{mg} / \mathrm{dL}$ or specific treatment, and (5) HDL-C $\leq 40 \mathrm{mg} / \mathrm{dL}$ in men and $\leq 50 \mathrm{mg} / \mathrm{dL}$ in women.

A dummy variable with four categories for all the possible permutations of CKD and MetS was created. The categories were as follows: (a) no CKD and no MetS $\left(\mathrm{CKD}^{-} / \mathrm{MetS}^{-}\right)$, (b) $\mathrm{CKD}$ and no $\mathrm{MetS}\left(\mathrm{CKD}^{+} / \mathrm{MetS}^{-}\right)$, (c) no $\mathrm{CKD}$ and MetS $\left(\mathrm{CKD}^{-} / \mathrm{MetS}^{+}\right)$, and $(\mathrm{d})$ both $\mathrm{CKD}$ and MetS $\left(\mathrm{CKD}^{+} / \mathrm{MetS}^{+}\right)$.

2.3. Cardiovascular Events. A detailed description of events and the process of adjudication can be found at the MESA website (http://www.mesa-nhlbi.org). Briefly, participants were contacted every 9-12 months to inquire about hospital admissions, cardiovascular diagnoses, and deaths. Hospital records were abstracted for possible CVD events and were sent for review and classification by an independent adjudication committee. For the purposes of this study, a 
CVD event was defined as incident myocardial infarction, resuscitated cardiac arrest, definite angina, probable angina if followed by revascularization, stroke, stroke death, coronary heart disease (CHD) death, other atherosclerotic death, and other CVD death as defined by the MESA protocol.

2.4. Statistical Analysis. Descriptive analyses of all the variables utilized in the data analysis were conducted. The baseline features were compared, using ANOVA or KruskalWallis tests for continuous variables and the chi-square or the Fisher exact tests for categorical variables, into four columns: Neither CKD/MetS; CKD only; MetS only; both CKD and MetS.

A Cox proportional hazards regression with and without adjustment for age, ethnicity, gender, study site, education, income, smoking, alcohol use, physical activity, total, and LDL cholesterol was performed to assess the independent association of CKD and MetS with incident CVD events with CKD and MetS in the same model.

We next divided the participants into four groups according to the presence/absence of CKD and/or MetS. Survival analysis was performed using cumulative eventfree Kaplan-Meier curves according to the presence/absence of CKD or MetS, and the groups were compared by logrank test for trend. A similar Cox proportional hazards regression was performed to investigate the relationship of the four groups (neither CKD/MetS, CKD only, MetS only, nor both CKD and MetS) with incident CVD events using two sets of models: unadjusted models and models adjusted (Table 2) for established cardiovascular risk factors (age, ethnicity, gender, study site, education, income, smoking, alcohol use, physical activity, and total and LDL cholesterol) using $\mathrm{CKD}^{-} / \mathrm{MetS}^{-}$as the reference category. The potential confounders were selected based on their relationship with cardiovascular disease and the prior literature. Additionally, a similar analysis was performed after excluding participants with prevalent diabetes.

Formal tests for additive and multiplicative interactions between CKD and MetS and prediction of incident CVD were also performed. We tested additive and multiplicative interactions in the proportional hazards model. A formal interaction term $\mathrm{CKD} \times$ MetS was introduced in the model with all covariates to test for multiplicative interaction.

Formal tests for indices of additive interaction such as relative excess risk due to interaction (RERI), attributable portion (AP), and synergy index (SI) were performed as described by Li and Chambless [28]. RERI is calculated as ((HR (both CKD and MetS) - HR (CKD alone) - HR (MetS alone) +1$)$ ). AP is calculated as RERI divided by HR (both CKD and MetS). SI is ratio of increase in hazard due to both exposures (CKD and MetS) to the sum of the increases due to one exposure alone. Please see The appendix for formal calculations. All statistical analyses were performed using JMP Version 8 (SAS Institute Inc., Cary, NC/USA).

\section{Results}

3.1. Participant Characteristics. The sociodemographic characteristics of the study sample are depicted in Table 1. The mean age of the sample was 61.6 years with the mean age being much higher at 69.7 years for participants with both CKD and MetS. A larger proportion of Chinese-Americans had no CKD/MetS $(81 \%)$ compared to other ethnicities, and only $1 \%$ of Chinese-Americans had both the conditions compared to $3 \%$ for all other ethnicities. Among women, $31 \%$ had MetS as compared to $26 \%$ of men. Diabetes was more prevalent in the groups with MetS and both $\mathrm{CKD} / \mathrm{MetS}$ compared to those without MetS or CKD/MetS. Smoking rates were not much different between the groups, whereas alcohol consumption was significantly higher in healthy participants. Additionally, the healthy participants were significantly more physically active as compared to participants with either or both conditions.

3.2. Cardiovascular Events. During 5.5 years of followup, $283 \mathrm{CVD}$ events were identified $\left(118, \mathrm{CKD}^{-} / \mathrm{MetS}^{-}\right.$group; $10, \mathrm{CKD}^{+} / \mathrm{MetS}^{-}$group; 120, $\mathrm{CKD}^{-} / \mathrm{MetS}^{+}$group; 35, $\mathrm{CKD}^{+} / \mathrm{MetS}^{+}$group). A Kaplan-Meier survival curve shows decreased survival free of CVD events across the four groups of CKD and MetS with a log test for trend which is statistically significant $(P<0.0001)$ (Figure 1$)$. These curves show significantly poorer survival in the $\mathrm{CKD}^{+} / \mathrm{MetS}^{+}$group.

When CKD and MetS were entered into the same model, the results of multivariate Cox regression analysis including age, sex, ethnicity, smoking habit, cholesterol both total and LDL-C, alcohol consumption, and physical activity found that CKD (HR 2.02, 95\% CI 1.43-2.79, $P<0.0001)$ and MetS (HR 2.55, 95\% CI 2.01-3.25, $P<0.0001$ ) were both significantly associated with incident CVD events.

Table 2 shows the results from a series of crude and multivariate regression analysis, showing how the association of CKD and MetS with CVD risk changed as groups of CVD risk factors were added to the regression model. In the crude model, the risk for CVD was significantly higher in the $\mathrm{CKD}^{+} / \mathrm{MetS}^{+}$group compared with the $\mathrm{MetS}^{-} / \mathrm{CKD}^{-}$ group (HR 8.46). The hazard in the $\mathrm{CKD}^{+} / \mathrm{MetS}^{+}$group remained highly significant in the multivariate model (HR 5.56). It remained significant even after further adjustment for antihypertensive medications and systolic blood pressure (HR 4.55, 95\% CI 3.01-6.73). Furthermore, when compared with the $\mathrm{CKD}^{+} / \mathrm{MetS}^{-}$group or with the $\mathrm{CKD}^{-} / \mathrm{MetS}^{+}$ group, the risk of CVD events was significantly higher in the $\mathrm{CKD}^{+} / \mathrm{MetS}^{+}$group in univariate Cox regression analysis (versus $\mathrm{CKD}^{+} / \mathrm{MetS}^{-}$group: HR 3.14, 95\% CI 1.61-6.69, $P<0.001$; versus $\mathrm{CKD}^{-} / \mathrm{MetS}^{+}$group: HR $3.38,95 \% \mathrm{CI}$ 2.28-4.87, $P=0.0001$ ) and in multivariate Cox regression analysis (versus $\mathrm{CKD}^{+} / \mathrm{MetS}^{-}$group: HR 3.89, 95\% CI 1.998.31; versus $\mathrm{CKD}^{-} / \mathrm{MetS}^{+}$group: $\mathrm{HR} 2.32$, 95\% CI $1.56-$ 3.37, $P<0.0001$, resp.).

We performed several additional analyses to address the robustness of these findings. Because patients with diabetes were more frequent in the $\mathrm{CKD}^{+} / \mathrm{MetS}^{+}$group, we repeated our analysis for the 4,591 participants without previous diabetes. In this study, 210 CVD events occurred during the follow-up period. The independent predictive value of $\mathrm{CKD}^{+} / \mathrm{MetS}^{+}$for $\mathrm{CVD}$ events was also confirmed by the Kaplan-Meier method (log rank test for trend chi-square = $60 ; P<0.0001)$ and by multivariate Cox regression analysis. 
TABLE 1: Baseline characteristics of study participants in the MESA cohort at baseline (2000).

\begin{tabular}{|c|c|c|c|c|c|c|}
\hline Variables & Total & $\mathrm{CKD}^{-} / \mathrm{MetS}^{-}$ & $\mathrm{CKD}^{+} / \mathrm{MetS}^{-}$ & $\mathrm{CKD}^{-} / \mathrm{MetS}^{+}$ & $\mathrm{CKD}^{+} / \mathrm{MetS}^{+}$ & $P$ value \\
\hline$n$ & 5163 & 3444 & 119 & 1455 & 145 & \\
\hline Age, years & $61.6(10.1)$ & $60.7(10.1)$ & $71.1(9.7)$ & $62.2(9.6)$ & $69.7(9.3)$ & 0.0001 \\
\hline Caucasian & $2283(44 \%)$ & $1565(69 \%)$ & $65(3 \%)$ & $581(25 \%)$ & $72(3 \%)$ & 0.0002 \\
\hline Chinese & $363(7 \%)$ & $293(81 \%)$ & $5(1 \%)$ & $61(17 \%)$ & $4(1 \%)$ & 0.0001 \\
\hline African & $1449(28 \%)$ & $953(66 \%)$ & $29(2 \%)$ & $428(30 \%)$ & $39(3 \%)$ & 0.0001 \\
\hline Hispanic & $1068(21 \%)$ & $633(59 \%)$ & $20(2 \%)$ & $385(36 \%)$ & $30(3 \%)$ & 0.0001 \\
\hline Male, \% & $2722(53 \%)$ & $1872(69 \%)$ & $74(3 \%)$ & $697(26 \%)$ & $79(3 \%)$ & 0.0001 \\
\hline Female, $\%$ & $2441(47 \%)$ & $1572(64 \%)$ & $45(2 \%)$ & $758(31 \%)$ & $66(3 \%)$ & 0.0001 \\
\hline DM, \% & $572(11 \%)$ & $128(4 \%)$ & $2(2 \%)$ & $394(27 \%)$ & $48(33 \%)$ & 0.0001 \\
\hline Current smokers, $\%$ & $741(14 \%)$ & $491(14 \%)$ & $19(16 \%)$ & $213(15 \%)$ & $18(12 \%)$ & 0.8 \\
\hline Current drinking, $\%$ & $3576(69 \%)$ & $2494(72 \%)$ & $78(66 \%)$ & $919(63 \%)$ & $85(59 \%)$ & 0.0001 \\
\hline Physical activity, min/wk & $1644(2395)$ & $1777(2537)$ & $1670(2855)$ & $1372(2012)$ & $1205(1683)$ & 0.0001 \\
\hline Total cholesterol, mg/dL & $193(35)$ & $194(34)$ & $187(38)$ & $193(36)$ & $188(39)$ & 0.08 \\
\hline $\mathrm{SBP}, \mathrm{mmHg}$ & $126(21)$ & $122(20)$ & $130(23)$ & $133(21)$ & $137(25)$ & 0.0001 \\
\hline DBP, $\mathrm{mmHg}$ & $72(10)$ & $72(10)$ & $71(10)$ & $74(10)$ & $72(11)$ & 0.0001 \\
\hline $\mathrm{LDL}-\mathrm{C}, \mathrm{mg} / \mathrm{dL}$ & $117(31)$ & $118(31)$ & $113(32)$ & $116(33)$ & $113(31)$ & 0.01 \\
\hline HDL-C, mg/dL & $51(15)$ & $55(15)$ & $52(14)$ & $43(10)$ & $42(11)$ & 0.0001 \\
\hline $\mathrm{TG}, \mathrm{mg} / \mathrm{dL}$ & $125(65)$ & $103(49)$ & $109(43)$ & $173(72)$ & $170(67)$ & 0.0001 \\
\hline Fasting glucose, $\mathrm{mmHg}$ & $96(27)$ & $90(19)$ & $89(9)$ & $110(40)$ & $109(33)$ & 0.0001 \\
\hline $\mathrm{eGFR}_{\mathrm{cys}}, \mathrm{mL} / \mathrm{min} / 1.73 \mathrm{~m}^{2}$ & $93(22)$ & $98(20)$ & $51(9)$ & $90(19)$ & $49(10)$ & 0.0001 \\
\hline \multicolumn{7}{|l|}{ MetS components, $\%$} \\
\hline HTN, \% & $2219(43 \%)$ & $1078(31 \%)$ & $70(59 \%)$ & $954(66 \%)$ & $117(81 \%)$ & 0.0001 \\
\hline Obesity, \% & $2763(54 \%)$ & $1319(38 \%)$ & $57(48 \%)$ & $1259(87 \%)$ & $128(88 \%)$ & 0.0001 \\
\hline Elevated TG, \% & $1457(28 \%)$ & $415(12 \%)$ & $13(11 \%)$ & $939(66 \%)$ & $90(62 \%)$ & 0.0001 \\
\hline Low HDL-C, \% & $1957(38 \%)$ & $736(21 \%)$ & $32(27 \%)$ & $1078(74 \%)$ & $111(77 \%)$ & 0.0001 \\
\hline Impaired Glucose, $\%$ & $2062(40 \%)$ & $1037(30 \%)$ & $39(33 \%)$ & $892(61 \%)$ & $94(65 \%)$ & 0.0001 \\
\hline CVD events, \% & $283(5 \%)$ & $118(3 \%)$ & $10(8 \%)$ & $120(8 \%)$ & $35(24 \%)$ & 0.0001 \\
\hline
\end{tabular}

$P$ values obtained by one aay analysis of variance. Data presented in total numbers (percentages) and continuous measures presented as mean value (standard deviation). DM: diabetes mellitus; SBP: dystolic blood pressure, mmHg; DBP: diastolic blood pressure, mmHg; LDL-C: mg/dL low density lipoprotein cholesterol; HDL-C: $\mathrm{mg} / \mathrm{dL}$ high density lipoprotein cholesterol; TG: $\mathrm{mg} / \mathrm{dL}$, triglyceride; $\mathrm{eGFR}_{\mathrm{cys} \mathrm{C}} \mathrm{mL} / \mathrm{min} / 1.73 \mathrm{~m}^{2}$ glomerular filtration rate estimated from cystatin C; CVD: cardiovascular events; MetS: metabolic syndrome; CKD: chronic kidney disease.

TABLE 2: Unadjusted and multivariate-adjusted HRs of CVD events associated with CKD and MetS.

\begin{tabular}{lcccc}
\hline & \multicolumn{2}{c}{ Total participants $(n=5,163)$} & \multicolumn{2}{c}{ Participants without diabetes $(n=4,591)$} \\
& Crude & Adjusted & Crude & Adjusted \\
\hline $\mathrm{CKD}^{-} / \mathrm{MetS}^{-}$ & 1 (Reference) & 1 (Reference) & 1 (Reference) & $1(\mathrm{Reference)}$ \\
\hline $\mathrm{CKD}^{+} / \mathrm{MetS}^{-}$ & $2.70(1.32-4.88)$ & $1.43(0.70-2.62)$ & $2.90(1.42-5.26)$ & $1.41(0.69-2.61)$ \\
\hline $\mathrm{CKD}^{-} / \mathrm{MetS}^{+}$ & $2.51(1.94-3.23)$ & $2.40(1.85-3.11)$ & $2.17(1.60-2.91)$ & $2.08(1.54-2.81)$ \\
\hline $\mathrm{CKD}^{+} / \mathrm{MetS}^{+}$ & $8.46(5.72-12.20)$ & $5.56(3.72-8.12)$ & $7.27(4.33-11.54)$ & $4.43(2.60-7.15)$ \\
\hline
\end{tabular}

Hazard ratios (95\% CI) adjusted for age, ethnicity, gender, study site, education, income, smoking, alcohol use, physical activity, and total and LDL cholesterol. HR: hazard ratio; CI: confidence interval; CVD: cardiovascular events; CKD: chronic kidney disease; MetS: metabolic syndrome.

Furthermore, even when compared with the $\mathrm{CKD}^{+} / \mathrm{MetS}^{-}$ group or with the $\mathrm{CKD}^{-} / \mathrm{MetS}^{+}$group, the risk of $\mathrm{CVD}$ events was significantly higher in the $\mathrm{CKD}^{+} / \mathrm{MetS}^{+}$group in the multivariate model (versus $\mathrm{CKD}^{+} / \mathrm{MetS}^{-}$group: $\mathrm{HR}$ 3.13, 95\% CI 1.48-7.03, $P=0.03$; versus $\mathrm{CKD}^{-} / \mathrm{MetS}^{+}$ group: HR 2.13, 95\% CI 1.23-3.49, $P=0.01$ ).

Finally, when interaction was tested between CKD and MetS, no multiplicative interaction was demonstrated
$(\mathrm{CKD} \times$ MetS, $P=0.2)$. When formal tests for additive interaction such as relative excess risk due to interaction (RERI), attributable portion (AP), and synergy index (SI) were performed, there was presence of significant additive interaction as shown in Table 3 and Appendix. RERI (95\% CI) was estimated at $2.73(0.57-4.85, P=0.02)$, AP (95\% CI) was estimated at $0.49(0.24-0.74)$, and SI $(95 \%$ CI) was estimated at $2.49(1.24-4.98)$. According to the 


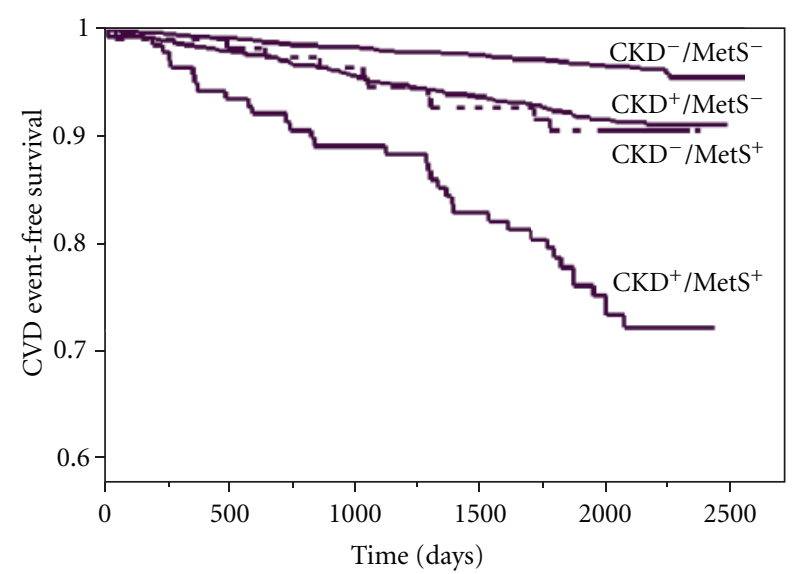

Figure 1: Kaplan-Meier plots showing cumulative CVD eventfree survival in participants in four groups divided by presence or absence of CKD and presence or absence of MetS (log-rank test for trend $\left.X^{2}=114 ; P<0.001\right)$; CKD: chronic kidney disease; MetS: metabolic syndrome; CVD: cardiovascular disease.

three measures of additive interaction between CKD and MetS, there is 2.73 relative excess risk due to the additive interaction, the risk of CVD in individuals who had been exposed to both risk factors (CKD and MetS) is 2.49 times higher than the sum of risks in individuals exposed to a single risk factor alone, and $49 \%$ of the incident CVD in individuals exposed to both risk factors is attributable to the additive interaction.

\section{Discussion}

In this ethnically diverse population of 5,163 individuals, aged 44-84, both chronic kidney disease and metabolic syndrome are independent predictors of incident cardiovascular events. This study identified a significant positive relationship between the cooccurrence of CKD and MetS and risk for CVD events. In a multivariate model, the hazard for incident cardiovascular events was increased due to presence of significant additive interaction between CKD and MetS. However, no multiplicative interaction between CKD and MetS was demonstrated. Additionally, the presence of both CKD and MetS conferred a significantly higher hazard compared to the presence of each condition separately. From the viewpoint of prevention and clinical practice, additive interaction is more important than multiplicative interaction, as it relates to a higher absolute excess of cases.

Defined by cystatin C, CKD $[7,29]$ has been shown to predict cardiovascular events and mortality in several studies. Ix et al. [7] studied 990 participants in the Heart and Soul Study and demonstrated that compared to participants in the lowest cystatin $\mathrm{C}$ quartile, those in the highest quartile (those with CKD) were at increased risk of cardiovascular events (HR, 2.0; 95\% CI, 1.0-3.8). Similarly, Deo et al. [29] studied 3,044 older adults ages from 70 to 79 over 6 years in the Health $\mathrm{ABC}$ cohort and found that those with CKD had significantly higher risk for cardiovascular death (HR, 2.24; 95\% CI, 1.30-3.86) compared to those without CKD. This
TABLE 3: Estimates of multiplicative and additive interaction (95\% confidence interval, CI) controlling for covariates.

\begin{tabular}{lccc}
\hline Parameters & Estimate & $\begin{array}{c}\text { CKD } \times \text { MetS } \\
95 \% \text { CI }\end{array}$ & $P$-value \\
\hline$\beta_{3}$ & 0.48 & $-0.23,1.28$ & 0.2 \\
RERI & 2.73 & $0.57,4.85$ & 0.02 \\
AP & 0.49 & $0.24,0.74$ & \\
SI & 2.49 & $1.24,4.98$ & \\
\hline
\end{tabular}

CKD: chronic kidney disease; MetS: metabolic syndrome; $\beta_{3}$ : parameter estimate of CKD $\times$ MetS testing for multiplicative interaction; RERI: relative excess risk due to additive interaction; AP: attributable portion; SI: synergy index are measures testing for additive interaction.

is similar to our findings where participants with $\mathrm{CKD}$, as defined by eGFR $\mathrm{CysC}_{\text {cy }} \leq 60 \mathrm{~mL} / \mathrm{min} / 1.73 \mathrm{~m}^{2}$ were at increased risk for CVD events (HR, 2.02, 95\% CI, 1.43-2.79) in a multivariate analysis. However, when the CKD only group was compared to participants with no CKD and no MetS, the hazard for CVD events was statistically insignificant, which is a reflection of low statistical power (Table 2).

Similarly, MetS has been shown to predict CVD events and mortality [11]. A meta-analysis involving 43 cohorts consisting of 172,573 individuals showed that MetS had a relative risk of cardiovascular events and death of 1.78 (95\% CI, 1.58-2.00). In our study, presence of MetS was an independent predictor of CVD events, and when the MetS only group was compared to the group with no $\mathrm{CKD} /$ no MetS, the hazard for CVD events remained statistically significant (Table 2).

Multiple studies [2-4] document the associations between CKD and MetS, and now mechanisms have been postulated that link the two conditions to each other. Recent findings, suggesting vitamin D being a modulator of both insulin resistance and the renin-angiotensin system [15] and the renin-angiotensin system in local pancreatic islet structure and function [16], suggest that perhaps renal dysfunction and MetS may share common pathological pathways. Clinically, it is seen that individuals with CKD have abnormalities in serum lipid concentrations and distribution (elevated triglycerides and lower high-density lipoprotein), diabetes mellitus, and hypertension $[2,12,13]$ which are also part of the MetS construct. Additionally, it has been proposed that the presence of both conditions leads to increased inflammation and oxidative stress, increased total peripheral resistance, and impaired left ventricular relaxation which increases the risk for CVD events [30]. This interplay of risk factors and pathological mechanisms implies that perhaps the cooccurrence of CKD and MetS identifies a group of individuals at higher risk for cardiovascular events.

A few studies $[30,31]$ document the role of these two conditions together as it relates to CVD events. Martin et al. [31] studied 13,115 individuals aged $\geq 35$ years from the NHANES III survey and found that the coclustering of CKD and MetS led to a significantly higher hazard for CVD mortality (HR, 3.23; 95\% CI, 2.56-3.70) when compared with individuals with no CKD and no MetS. Similarly, Iwashima et al. [30] studied 1,160 essential hypertensive 
individuals for a mean period of 4.8 years and found that the presence of both CKD and MetS conferred a higher risk for CVD events (HR, 3.58; 95\% CI, 2.14-5.95) compared to the no $\mathrm{CKD} /$ no MetS group. Our findings are a validation and extension of these findings in a multiethnic cohort free of cardiovascular disease at baseline. In contrast to the study by Martins, we found no significant association in the CKD only group but found significant association in the MetS only group which is perhaps due to small numbers of CKD only individuals. Second, our study included a cohort with both with and without hypertension.

Our study has several limitations. First, MESA did not directly measure glomerular filtration rate (GFR); therefore, we cannot be certain that the association between elevated cystatin C level and CVD events are solely caused by its approximation of impaired GFR. This approach can lead to misclassification of individuals due to biased estimates. Second, although efforts were made to adjust for known confounders, there remains a possibility of failure to adjust for unknown confounders or inadequate adjustment of established risk factors (severity and duration of hypertension, diabetes) resulting in spurious results due to residual confounding. Third, some studies suggest that corticosteroids [32] and thyroid function [33] are associated with cystatin $\mathrm{C}$, and since adjustment with these measures was not performed, results should be interpreted with caution in this subset of individuals. The distribution of metabolic syndrome components may vary from population to population, which may have an impact on the external validity of findings if the joint association/interaction is mostly due to one of the components. The results of the study are limited to individuals without cardiovascular disease and may not be generalizable to a population with known coronary artery disease due to selection bias. Also, due to the cross-sectional nature of the risk factors, the association between CKD, MetS, and CVD events could potentially be due to post assessment residual confounding.

\section{Conclusion}

This study shows that the co-occurrence of CKD and MetS results in an increased hazard for cardiovascular events in a multiethnic population. Although, no multiplicative interaction was demonstrated, there is significant presence of additive interaction. Both CKD and MetS are independent predictors of CVD but their combination furthers the risk independent of conventional risk factors. From the clinical viewpoint, physicians should become more cognizant that concomitant CKD and MetS lead to increased risk for CVD events. Additionally, assessment of renal function in individuals with MetS and vice versa may lead to improved risk stratification for cardiovascular disease in clinical practice. More studies are needed in the future to explore the temporal relationship between CKD, MetS and cardiovascular disease. Additionally, studies are needed to explore whether novel and aggressive pharmacological and behavioral modifications in individuals with both CKD and MetS, will lead to reduction in CVD risk.

\section{Disclosures}

The authors had full access to the data and take responsibility for the integrity of the data. All authors have read and agree to the study as written.

\section{Appendix}

Using the output from Tables 4 and 5, the calculations of RERI and the test for the additive interaction are illustrated below.

(1) Relative Excess Risk due to Interaction (RERI) = $\mathrm{HR}_{11}-\mathrm{HR}_{10}-\mathrm{HR}_{01}+1$, where $\mathrm{HR}_{11}$ is hazard due to both CKD and MetS, $\mathrm{HR}_{10}$ is hazard due to CKD alone, and $\mathrm{HR}_{01}$ is hazard due to MetS alone.

$$
\begin{aligned}
\text { RERI }= & \mathrm{e}^{\beta 1+\beta 2+\beta 3}-\mathrm{e}^{\beta 1}-\mathrm{e}^{\beta 2}+1 \\
= & 5.56-1.43-2.40+1=2.73, \\
\operatorname{VAR}(\operatorname{RERI})= & a_{2}^{1} \times \operatorname{var} \beta_{1}+a_{2}^{2} \times \operatorname{var} \beta_{2}+a_{3}^{2} \times \operatorname{var} \beta_{3} \\
& +2\left(a_{1} a_{2} \times \operatorname{Cov}\left(\beta_{1} \beta_{2}\right)+a_{1} a_{3}\right. \\
& \left.\times \operatorname{Cov}\left(\beta_{1} \beta_{3}\right)+a_{2} a_{3} \times \operatorname{Cov}\left(\beta_{2} \beta_{3}\right)\right), \\
a_{1}= & \mathrm{e}^{\beta 1+\beta 2+\beta 3}-\mathrm{e}^{\beta 1} \\
= & 5.56-1.43=4.13, \\
a_{2}= & \mathrm{e}^{\beta 1+\beta 2+\beta 3}-\mathrm{e}^{\beta 2} \\
= & 5.56-2.40=3.16, \\
a_{3}= & \mathrm{e}^{\beta 1+\beta 2+\beta 3}=5.56 .
\end{aligned}
$$

$\operatorname{VAR}($ RERI $)=1.19$, SE $($ RERI $)=1.09$. Hence, $t=$ RERI $/$ VAR $($ RERI $)=2.73 / 1.19=2.28, P=0.02$, and a 95\% confidence interval estimate for RERI, RERI $\pm 1.96 \times \mathrm{SE}(\mathrm{RERI})=(0.57$ $-4.85)$ :

(2) Attributable portion $(\mathrm{AP})=\left(\mathrm{e}^{\beta 1+\beta 2+\beta 3}-\mathrm{e}^{\beta 1}-e^{\beta 2}+\right.$ 1) $/\left(\mathrm{e}^{\beta 1+\beta 2+\beta 3}\right)=2.73 / 5.56=0.49$ :

$$
\begin{aligned}
& a_{1}=\frac{e^{\beta 2-1}}{e^{\beta 1+\beta 2+\beta 3}}, \\
& a_{2}=\frac{e^{\beta 1-1}}{e^{\beta 1+\beta 2+\beta 3}}, \\
& a_{3}=\frac{e^{\beta 1+\beta 2-1}}{e^{\beta 1+\beta 2+\beta 3}} .
\end{aligned}
$$

$\operatorname{VAR}(\mathrm{AP})=0.016, \mathrm{SE}(\mathrm{AP})=0.126$. Hence, $95 \%$ confidence interval estimate for $\mathrm{AP}, \mathrm{AP} \pm 1.96 \times \mathrm{SE}(\mathrm{AP})=(0.24-0.74)$.

(3) Synergy index (SI) $=e^{\beta 1+\beta 2+\beta 3}-1 / \mathrm{e}^{\beta 1+\beta 2}-2=$ $4.56 / 1.83=2.49$.

$$
\begin{aligned}
& a_{1}=a_{3}-\left(\frac{\mathrm{e}^{\beta 1}}{\mathrm{e}^{\beta 1+\beta 2}}-2\right), \\
& a_{2}=a_{3}-\left(\frac{\mathrm{e}^{\beta 2}}{\mathrm{e}^{\beta 1+\beta 2}}-2\right), \\
& a_{3}=\left(\frac{\mathrm{e}^{\beta 1+\beta 2+\beta 3}}{\mathrm{e}^{\beta 1+\beta 2+\beta 3}}-1\right) .
\end{aligned}
$$


TABLE 4: Output from proportional hazards models.

\begin{tabular}{cccccccc}
\hline & Parameter & Estimated $\beta$ & SE $(\beta)$ & $t$-test & $P$ value & HR & 95\% CI \\
\hline \multirow{3}{*}{ Model $^{*}$} & CKD & 0.36 & 0.33 & 1.14 & 0.29 & 1.43 & $0.74-2.75$ \\
& MetS & 0.87 & 0.13 & 44.05 & 0.0001 & 2.40 & $1.85-3.10$ \\
& CKD $\times$ MetS & 0.48 & 0.38 & 1.61 & 0.2 & 1.62 & $0.77-3.44$ \\
\hline
\end{tabular}

* Parameter estimates and test statistics for interaction between CKD (chronic kidney disease) and MetS (metabolic syndrome) adjusted for covariates.

TABle 5: Covariance matrix of the set of $\beta$ coefficients from the proportional hazards models.

\begin{tabular}{lccc}
\hline & $\beta_{1}(\mathrm{CKD})$ & $\beta_{2}(\mathrm{MetS})$ & $\beta_{3}(\mathrm{CKD} \times \mathrm{MetS})$ \\
\hline$\beta_{1}(\mathrm{CKD})$ & 0.1119220903 & 0.0087980044 & -0.1097780898 \\
$\beta_{2}(\mathrm{Met} S)$ & 0.0087980044 & 0.0173595635 & -0.0170957203 \\
$\beta_{3}(\mathrm{CKD} \times$ MetS $)$ & -0.1097780898 & -0.0170957203 & 0.1463599444 \\
\hline
\end{tabular}

$\operatorname{VAR}(\log S)=0.125, \operatorname{SE}(\log S)=0.354$. Hence, $95 \%$ confidence interval estimate for SI, SI $\pm 1.96 \times \mathrm{SE}(\mathrm{SI})=(1.24-$ 4.98).

\section{Acknowledgments}

Timothy M. Morgan Ph.D., Professor of Biostatistics, Public Health Division, at Wake Forest University provided input in statistics. This research was supported by NHLBI, NIH T32 training grant (2 T32 HL 076132-06 A1), and contracts N01-HC-95159 through N01-HC-95169 from the National Heart, Lung, and Blood Institute. The authors thank the other investigators, the staff, and the participants of the MESA study for their valuable contributions. A full list of participating MESA investigators and institutions can be found at http://www.mesa-nhlbi.org/.

\section{References}

[1] J. Coresh, D. Byrd-Holt, B. C. Astor et al., "Chronic kidney disease awareness, prevalence, and trends among U.S. adults, 1999 to 2000," Journal of the American Society of Nephrology, vol. 16, no. 1, pp. 180-188, 2005.

[2] J. Chen, P. Muntner, L. L. Hamm et al., "The metabolic syndrome and chronic kidney disease in U.S. adults," Annals of Internal Medicine, vol. 140, no. 3, pp. 167-I39, 2004.

[3] M. Kurella, J. C. Lo, and G. M. Chertow, "Metabolic syndrome and the risk for chronic kidney disease among nondiabetic adults," Journal of the American Society of Nephrology, vol. 16, no. 7, pp. 2134-2140, 2005.

[4] M. P. Alexander, T. V. Patel, Y. M. K. Farag, A. Florez, H. G. Rennke, and A. K. Singh, "Kidney pathological changes in metabolic syndrome: a cross-sectional study," American Journal of Kidney Diseases, vol. 53, no. 5, pp. 751-759, 2009.

[5] J. I. Cleeman, "Executive summary of the third report of the National Cholesterol Education Program (NCEP) expert panel on detection, evaluation, and treatment of high blood cholesterol in adults (adult treatment panel III)," Journal of the American Medical Association, vol. 285, no. 19, pp. 2486-2497, 2001.

[6] E. S. Ford, W. H. Giles, and W. H. Dietz, "Prevalence of the metabolic syndrome among US adults: findings from the Third National Health and Nutrition Examination Survey,"
Journal of the American Medical Association, vol. 287, no. 3, pp. 356-359, 2002.

[7] J. H. Ix, M. G. Shlipak, G. M. Chertow, and M. A. Whooley, "Association of cystatin $\mathrm{C}$ with mortality, cardiovascular events, and incident heart failure among persons with coronary heart disease: data from the Heart and Soul Study," Circulation, vol. 115, no. 2, pp. 173-179, 2007.

[8] A. S. Go, G. M. Chertow, D. Fan, C. E. McCulloch, and C. Y. Hsu, "Chronic kidney disease and the risks of death, cardiovascular events, and hospitalization," The New England Journal of Medicine, vol. 351, no. 13, pp. 1296-1370, 2004.

[9] W. Koenig, D. Twardella, H. Brenner, and D. Rothenbacher, "Plasma concentrations of cystatin $\mathrm{C}$ in patients with coronary heart disease and risk for secondary cardiovascular events: more than simply a marker of glomerular filtration rate," Clinical Chemistry, vol. 51, no. 2, pp. 321-327, 2005.

[10] H. M. Lakka, D. E. Laaksonen, T. A. Lakka et al., "The metabolic syndrome and total and cardiovascular disease mortality in middle-aged men," Journal of the American Medical Association, vol. 288, no. 21, pp. 2709-2716, 2002.

[11] A. S. Gami, B. J. Witt, D. E. Howard et al., "Metabolic syndrome and risk of incident cardiovascular events and death: a systematic review and meta-analysis of longitudinal studies," Journal of the American College of Cardiology, vol. 49, no. 4, pp. 403-414, 2007.

[12] M. G. Shlipak, L. F. Fried, M. Cushman et al., "Cardiovascular mortality risk in chronic kidney disease: comparison of traditional and novel risk factors," Journal of the American Medical Association, vol. 293, no. 14, pp. 1737-1745, 2005.

[13] R. N. Foley, C. Wang, and A. J. Collins, "Cardiovascular risk factor profiles and kidney function stage in the US general population: the NHANES III study," Mayo Clinic Proceedings, vol. 80, no. 10, pp. 1270-1277, 2005.

[14] K. C. Chiu, A. Chu, V. L. W. Go, and M. F. Saad, "Hypovitaminosis $\mathrm{D}$ is associated with insulin resistance and $\beta$ cell dysfunction," American Journal of Clinical Nutrition, vol. 79, no. 5, pp. 820-825, 2004.

[15] S. Williams, K. Malatesta, and K. Norris, "Vitamin D and chronic kidney disease," Ethnicity \& disease, vol. 19, no. 4, pp. S5-8, 2009.

[16] P. S. Leung and M. C. Chappell, "A local pancreatic reninangiotensin system: endocrine and exocrine roles," International Journal of Biochemistry and Cell Biology, vol. 35, no. 6, pp. 838-846, 2003.

[17] T. Lau, P. O. Carlsson, and P. S. Leung, "Evidence for a local angiotensin-generating system and dose-dependent inhibition 
of glucose-stimulated insulin release by angiotensin II in isolated pancreatic islets," Diabetologia, vol. 47, no. 2, pp. 240 248, 2004.

[18] A. Bökenkamp, M. Domanetzki, R. Zinck, G. Schumann, D. Byrd, and J. Brodehl, "Cystatin C-a new marker of glomerular filtration rate in children independent of age and height," Pediatrics, vol. 101, no. 5, pp. 875-881, 1998.

[19] D. J. Newman, H. Thakkar, R. G. Edwards et al., "Serum cystatin $\mathrm{C}$ measured by automated immunoassay: a more sensitive marker of changes in GFR than serum creatinine," Kidney International, vol. 47, no. 1, pp. 312-318, 1995.

[20] M. G. Shlipak, M. J. Sarnak, R. Katz et al., "Cystatin C and the risk of death and cardiovascular events among elderly persons," The New England Journal of Medicine, vol. 352, no. 20, pp. 2049-2060, 2005.

[21] M. G. Shlipak, M. J. Sarnak, R. Katz et al., "Cystatin-C and mortality in elderly persons with heart failure," Journal of the American College of Cardiology, vol. 45, no. 2, pp. 268-271, 2005.

[22] L. A. Stevens, J. Coresh, C. H. Schmid et al., "Estimating GFR using serum cystatin $\mathrm{C}$ alone and in combination with serum creatinine: a pooled analysis of 3,418 individuals with CKD," American Journal of Kidney Diseases, vol. 51, no. 3, pp. 395406, 2008.

[23] D. E. Bild, D. A. Bluemke, G. L. Burke et al., "Multi-ethnic study of atherosclerosis: objectives and design," American Journal of Epidemiology, vol. 156, no. 9, pp. 871-881, 2002.

[24] A. V. Chobanian, G. L. Bakris, H. R. Black et al., "Seventh report of the Joint National Committee on Prevention, Detection, Evaluation, and Treatment of High Blood Pressure," Hypertension, vol. 42, no. 6, pp. 1206-1252, 2003.

[25] W. T. Friedewald, R. I. Levy, and D. S. Fredrickson, "Estimation of the concentration of low-density lipoprotein cholesterol in plasma, without use of the preparative ultracentrifuge," Clinical Chemistry, vol. 18, no. 6, pp. 499-502, 1972.

[26] E. J. Erlandsen, E. Randers, and J. H. Kristensen, "Evaluation of the dade behring $\mathrm{N}$ latex cystatin $\mathrm{C}$ assay on the dade behring nephelometer II system," Scandinavian Journal of Clinical and Laboratory Investigation, vol. 59, no. 1, pp. 1-8, 1999.

[27] S. M. Grundy, J. I. Cleeman, S. R. Daniels et al., "Diagnosis and management of the metabolic syndrome: an American Heart Association/National Heart, Lung, and Blood Institute scientific statement," Circulation, vol. 112, no. 17, pp. 27352752, 2005.

[28] R. Li and L. Chambless, "Test for additive interaction in proportional hazards models," Annals of Epidemiology, vol. 17, no. 3, pp. 227-236, 2007.

[29] R. Deo, C. L. Wassel Fyr, L. F. Fried et al., "Kidney dysfunction and fatal cardiovascular disease-an association independent of atherosclerotic events: results from the Health, Aging, and Body Composition (Health ABC) study," American Heart Journal, vol. 155, no. 1, pp. 62-68, 2008.

[30] Y. Iwashima, T. Horio, K. Kamide et al., "Additive interaction of metabolic syndrome and chronic kidney disease on cardiac hypertrophy, and risk of cardiovascular disease in hypertension," American Journal of Hypertension, vol. 23, no. 3, pp. 290 298, 2010.

[31] D. Martins, C. Ani, D. Pan, O. Ogunyemi, and K. Norris, "Renal dysfunction, metabolic syndrome and cardiovascular disease mortality," Journal of Nutrition and Metabolism, vol. 2010, Article ID 167162, 8 pages, 2010.

[32] L. Risch, R. Herklotz, A. Blumberg, and A. R. Huber, "Effects of glucocorticoid immunosuppression on serum cystatin $\mathrm{C}$ concentrations in renal transplant patients," Clinical Chemistry, vol. 47, no. 11, pp. 2055-2059, 2001.

[33] P. Wiesli, B. Schwegler, G. A. Spinas, and C. Schmid, "Serum cystatin C is sensitive to small changes in thyroid function," Clinica Chimica Acta, vol. 338, no. 1-2, pp. 87-90, 2003. 


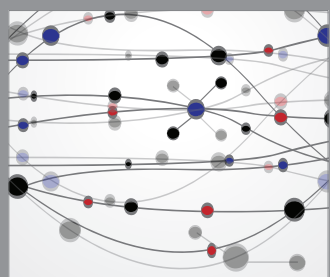

The Scientific World Journal
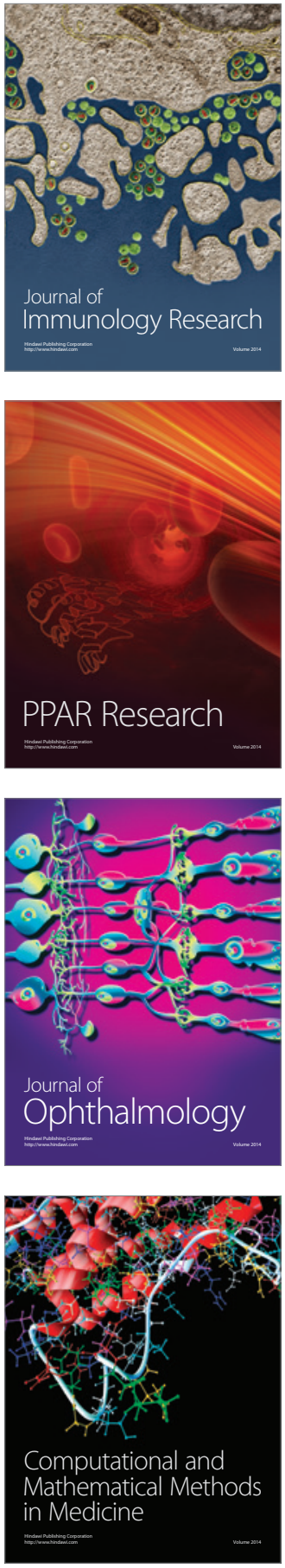

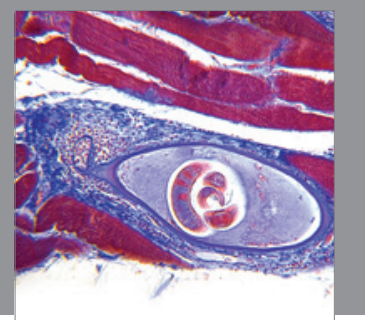

Gastroenterology

Research and Practice
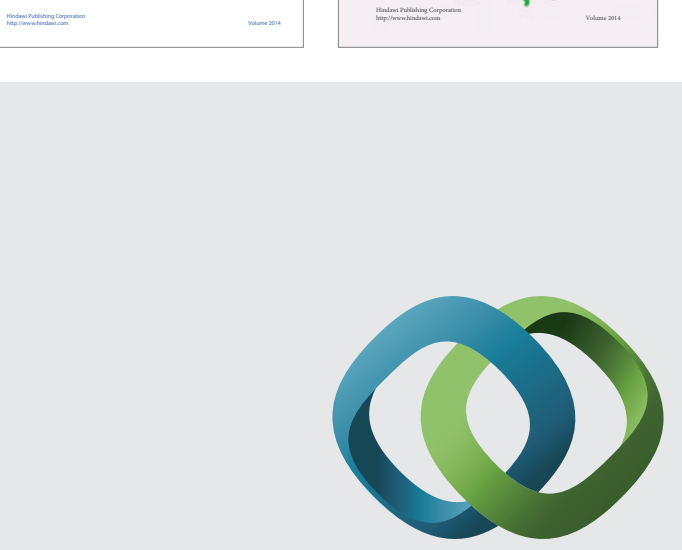

\section{Hindawi}

Submit your manuscripts at

http://www.hindawi.com
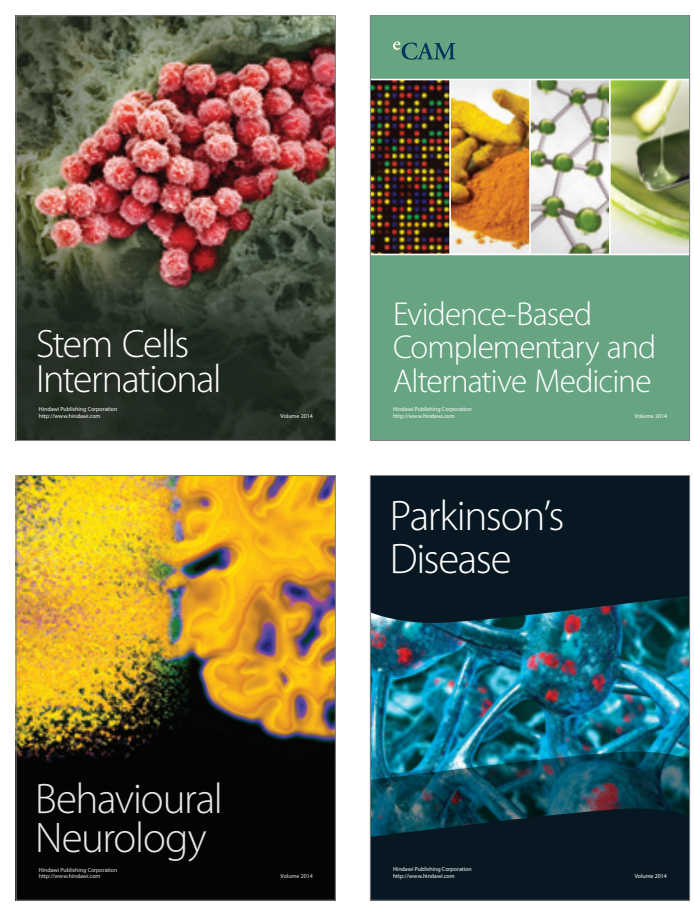

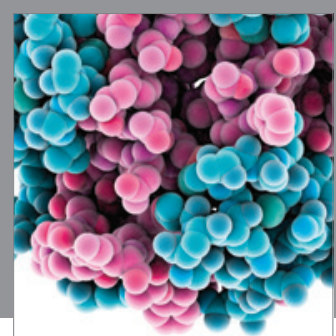

Journal of
Diabetes Research

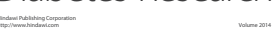

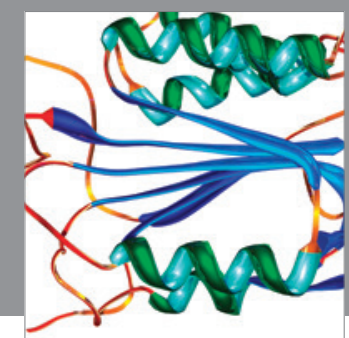

Disease Markers
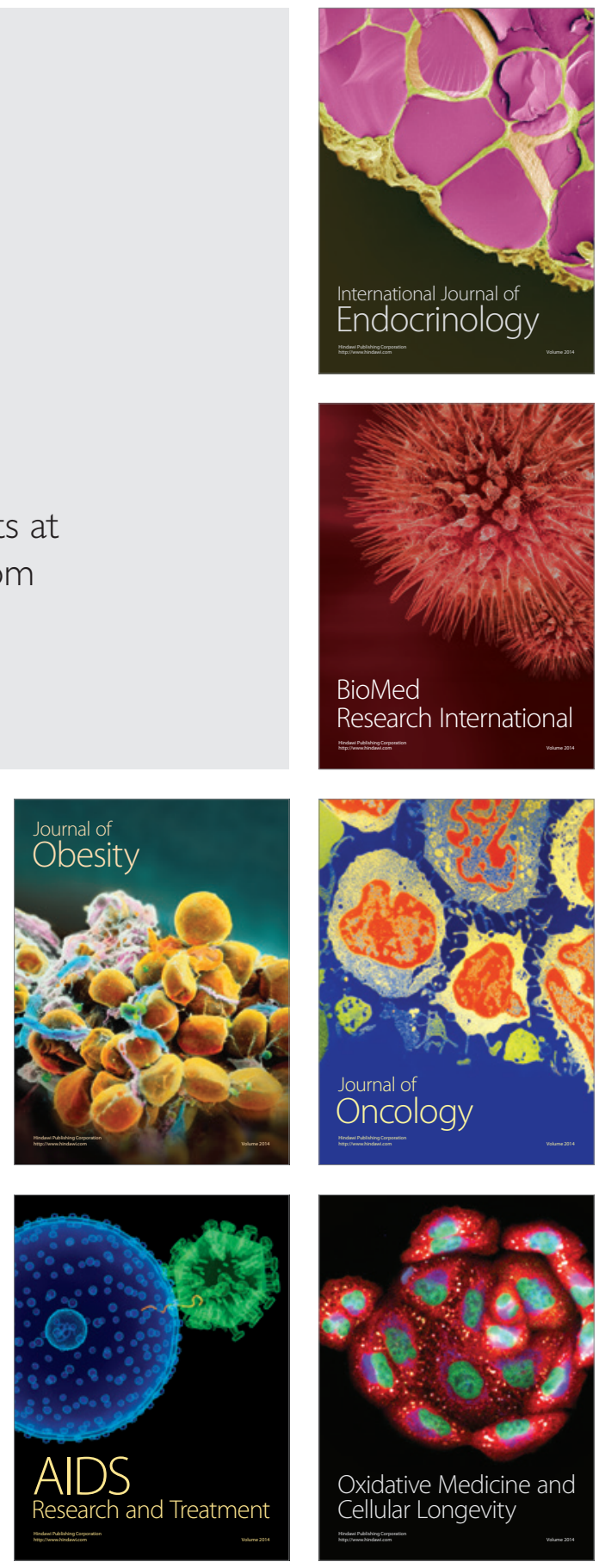